\title{
BCY 2 - Second Breast Cancer in Young Women Conference 4th-5th November 2014 Dublin, Ireland
}

Breast cancer (BC) in women under 40 years of age is a complex disease which strikes in a phase of life when young women are at the peak of their reproductive years and when family planning, professional career, and partnership have a lot of importance. Furthermore, as young women are more likely to have a risk of a higher grade and stage of the disease, tumor biology at the time of diagnosis and genetic factors play an important role, medical treatment and consultation has to be well adapted to the needs of this population. So far, little is known about the etiology of breast cancer in young women and more research is needed to establish an optimal management of these patients.

The Breast Cancer in Young Women Conference was initiated in 2012 by high-ranking breast cancer specialists from Europe, Israel, and the US to create a platform about novel research results, future research concepts and to give the opportunity of networking and discussion in this so far poorly researched field. Additionally, the first international consensus guidelines for breast cancer in young women were developed by international BC specialists, being finally published in June 2014 (Breast 2014;23:209-220).

Initially planned to take place in Tel Aviv, Israel, BCY 2 was changed this year to take place again in Dublin, Ireland because of the political conflicts in the Middle East. The BCY 2 conference was supported and organized by the European School of Oncology (ESO) (www.eso.net). In 9 sessions and 25 talks in total, all 'hot topics' around the treatment and diagnosis of young BC patients were covered. International speakers gave an overview of recent results of breast cancer research, newest developments in the treatment of young breast cancer patients and an outlook on the results to be presented in San Antonio in December 2014. During the conference, the 205 participants from 35 different countries heard talks that touched upon tumor biology and pathology, genetics, diagnostics, surgery, systemic therapy, radiotherapy, fertility preservation, and supportive offers.

After the presentation by Bella Kaufmann from the Israel Cancer Association (IL), one of the chairs of the conference, BCY 2 was opened by a field report of a young BC patient. With a very personal report, Nicola Elmer (IE) illustrated this intensive and difficult phase of her life around the $\mathrm{BC}$ diagnosis and all her problems during and after systemic therapy, so that a perfect introduction to the conference was given. Ann Partridge (US) continued in another keynote lecture to refer about the growing population of young $\mathrm{BC}$ patients, and its relevance for public health and the importance of improvement of psychosocial and medical care of those patients mainly focusing on the US perspective. She also focused on the unique aspects of young women with BC. They often present an advanced disease because of delays of diagnosis ('you are too young to have breast cancer') and a more aggressive tumor biology (e.g. ER negative, high grade, LVI disease, HER2-positive). Genetics, fertility and sexuality concerns as well as body image and the premenopausal situation play an important role in these patients, they need to be understood and supported.

Diagnostic tools were presented in the session by M. SklairLevy (IL), who discussed new technologies of breast imaging such as tomosynthesis (a modification of digital mammography to create 3-dimensional data), contrast-enhanced spectral mammography (CESM), automated breast volume scanner and also nuclear breast imaging (positron emission mammography, FDG) which are intended to be implemented in the individual diagnosis of young breast cancer patients.

The session on hereditary and familial breast cancer was well discussed. E. Levy-Lahad (IL) presented data about the right time for genetic testing and discussed issues about rapid genetic testing before initiating any treatment and traditional $B R C A$-testing in the young patient. As genetic testing has a yield of about $25 \%$ in the young patient, it affects treatment decisions and has therefore therapeutic impact. The goal of genetic testing might in any case be a treatment-focused genetic testing (TFGT), after diagnosis but before surgery. In her talk about local therapy considerations in BRCA1/2 mutation carriers, Ella Evron (IL) showed the markedly increased risk of $B R C A$ carriers of contralateral $\mathrm{BC}$ and presented the design of an initiated Israeli multicenter study on the prophylactic contralateral irradiation after breast conserving therapy as a potential option of prevention in high-risk patients and to reduce the rate of bilateral mastectomy. New systemic therapies in hereditary BC were discussed by Judy Garber who presented recent data with a neoadjuvant regimen of platinum in TNBC (pathologic complete remission (pCR) 61\%, Breast Cancer Res Treat 2014;147:401-405) as also shown by GEPARSIXTO (higher pCR rates after neoadjuvant anthracycline/taxane in TNBC). PARPP inhibitors such as olaparib (Olympia) or niraparib (BRAWO) for 1 year after neo-/adjuvant therapy are currently tested in several protocols as also in combination with platinum (carboplatin/gemcitabine/iniparib) in the PrECOG 0105 scheme.

\section{KARGER}

Fax +497614520714
() 2015 S. Karger GmbH, Freiburg

$1661-3791 / 15 / 0101-0055 \$ 39.50 / 0$ 
In the session about local-regional therapy by $\mathrm{O}$. Gentilini (IT), different surgical options for young women with $\mathrm{BC}$ and also $B R C A$ mutation carriers were discussed, that had become more important in the last years with an increasing demand for counseling ('Jolie effect'). As data about the surgical outcome are still missing, the surgical treatment should in general be the same as in the older patients while in the case of mastectomy immediate reconstruction should be offered.

The first day of BYC 2 closed with a special session of Deborah Fenlon (UK) about nursing in younger women with BC and the importance of the 'being there' for young patients and their unique needs and special nursing offers for these patients.

The second day of the conference was opened by K. Gelmon (US) about aspects of tumor biology. She once more emphasized the higher risk of relapse and worse outcome in younger women with $\mathrm{BC}$ and the still poorly understood difference of tumor biology and its therapy in that special population of patients.

In the session of systemic therapies, Sybille Loibl (DE) reported data of the NSABP-B18 and B-27 study, which showed a better disease free and overall survival for women $<50$ years after neoadjuvant instead of adjuvant chemotherapy. Additionally, young women seem to have a higher rate of pCR after being treated with an anthracyline and taxane based chemotherapy. In that context, Nadia Harbeck (DE) supplemented with data of GEPARSIXTO study which showed a higher pCR by adding weekly carboplatin to a neoadjuvant treatment in TNBC while in the metastatic situation the benefit of platinum is still discussed. As in the advanced TNBC, there is still a lack of international chemostandards. Professor Harbeck illustrated the importance of current clinical trials like the tnAcity trial (nab-paclitaxel/carboplatin versus nab-paclitaxel/gemcitabine versus carboplatin/gemcitabine) to establish a first-line standard in the treatment in the metastatic TNBC. In her talk about endocrine therapy, Olivia Pagani $(\mathrm{CH})$ gave an update of new endocrine regimens and presented recent data about the significant reduction of recurrences by the use of aromatase inhibitors plus ovarian function suppression (OFS) as a new option in premenopausal ( $\mathrm{HR}+$ ) patients. Also she gave an outlook on data about tamoxifen and OFS (SOFT and TEXT trial) which were later presented at San Antonio Breast Cancer Conference in December 2014. What can be discussed in premenopausal and high risk patients is an extended treatment of tamoxifen, considering the patient's life quality.

As one of the most important issues in young patients, fertility and concerns about family planning may play a very important role. Shani Paluch-Shimon and Hila Raanani, both from Israel, discussed those issues in the next session. Regarding fertility preservation, more experience could be made in the last years with ovarian tissue cryopreservation and transplantation (first successful pregnancy in 2005), also new regimens of stimulation before chemo have been developed. Thus, co-administration of tamoxifen during stimulation provides safety without affecting fertility preservation outcome and can be used in BC patients. More data is still needed to handle the desire to have a baby during a long-time therapy (endocrine therapy) from the oncological

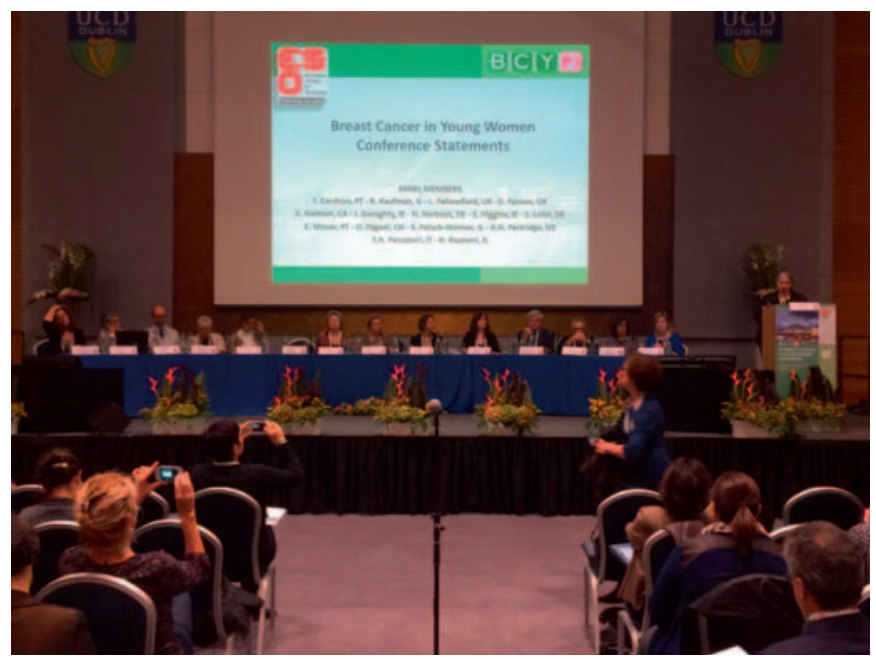

Panel at the BCY 2 conference, Dublin.

perspective. No data exist about the safety of interruption of the adjuvant therapy.

Before the poster session, presentation of the best abstracts took place. Thus, Efrat Dagan for research about PGD decisions, Matteo Lambertini from Italy with a prospective study about pregnancy and fertility, and Megan McCann from the US with the development of a toolkit approach for the unique needs of young BC patients were honored for their research.

In her talk about advanced breast cancer, Fatima Cardoso (PT), chair of the conference, pointed out once more the lack of proven standards in the therapy of young $\mathrm{BC}$ patients, especially for $B R C A$ mutations carriers, and that younger age alone is still no indication for a more aggressive therapy. Also she admitted that the young patient should undergo the same standards of diagnostic and therapy as the older patients with the same disease in metastatic BC.

The last session of the conference concerned survivorship and issues of quality of life. Ann Partridge (US) talked about the reality of oncobrain as a side and long-term effect of our therapies. So far, pharmaceutical agents have not been shown to be effective, settings for cognitive rehabilitation have to be implemented in the occupational therapy. Another important but little discussed issue is the awareness of achieving life quality after the treatment. There is a lack of survivorship care plans or post treatment navigators with recommendations for healthy behaviors, follow-up plans for the disease, or just access of medical and psychosocial support. E-resources like www.cancer.net/survivorship or www. ystourdepink.org are examples for such survivorship platforms.

The conference closed with a consensus statement of the leading international $\mathrm{BC}$ specialists to give the base for the second consensus guidelines for breast cancer in young women. The team of specialist consisted of Fatima Cardoso (PT), Bella Kaufmann (IL), Leslie Fallowfield (UK), Deborah Fenlon (UK), Karen Gelmon (CA), J Geraghty (IE), Nadia Harbeck (DE), S. Higgings (IE), Sybille Loibl (DE), Elizabeth Moser (PT), Olivia Pagani (CH), Shani Paluch-Shimon (IL), Ann Partridge (US), Fedro Peccatori (IT), and Hila Raanani (IL). 
In summary, the organizers of BCY 2 provided an excellent meeting on this very important topic by inviting the leading experts for clinical treatment and medical research in young breast cancer patient. With selected talks about all different aspects within the diagnosis and treatment of the young BC patient, the current issues were well reflected. It was emphasized that the hot topics in the support of young BC patients are around fertility, genetics and that our young patients thus differ significantly from postmenopausal patients what has to be well respected in the treatment of the young patient.
As this is the only meeting focused on the young BC patient, it renders an excellent platform for networking, discussion, and exchange of newest research results in a so far rarely discussed field and points out the importance of more research to create an optimal management in the treatment of young women with breast cancer. The next meeting will take place in 2016, more information can be found at www.eso.net.

M. Burgmann, R. Wuerstlein

Breast Center and CCC, University of Munich (LMU), Munich

\section{COMBATing Breast Cancer 2014: Molecular Understanding of Breast Cancer: Digging Deeper}

On November 21-22, 2014, the 7th Conference on Molecular Basics and Therapeutic Implications in Breast Cancer (COMBAT) was held in Düsseldorf, Germany. This year's conference followed the motto 'Molecular Understanding of Breast Cancer: Digging Deeper'. Under the chairmanship of Nadia Harbeck (University Hospital Munich), Achim Rody (University Hospital Schleswig-Holstein Campus Lübeck) and Michael Gnant (Medical University of Vienna), the conference was again an outstanding success. Mostly young clinicians and researchers from Germany, Austria, and Switzerland participated to share and further their insight into molecular biology and the scientific background of modern individualized breast cancer therapy. Moreover, national and international guests presented the latest discoveries and insights in breast cancer research in over 20 lectures covering a broad scientific spectrum ranging from conventional and targeted therapeutic agents to risk prediction and therapy selection based on multigene assays to practical and logistical aspects of breast cancer care.

\section{Combating Award}

The winner of the annual Combating Award was Frederik Marmé of Heidelberg University Hospital and the German National Center for Tumor Diseases (NCT Heidelberg) for his excellent continuous work in translational research in breast cancer. For his professorial thesis, he researched genetic polymorphisms and their establishment as prognostic and predictive markers in gynecological malignancies.

\section{AGO TraFo Meets COMBAT}

Continuing the successful partnership established in 2011, the AGO TraFo Meeting (AGO TraFo = Arbeitsgemeinschaft Gynäkologische Onkologie, Kommission Translationale Forschung) was again held in conjunction with the COMBAT Meeting. Translational research refers to the continuous exchange between clini- cians, clinical researchers, and lab-based scientists and the backand-forth of questions and answers to provide explanations for observed clinical phenomena and to evaluate scientific discoveries regarding their clinical use. Chaired by Tanja Fehm (University Hospital Düsseldorf), Peter Fasching (University Hospital Erlangen) and Dieter Niederacher (University Hospital Düsseldorf), the symposium started with the session on 'Predictive Markers and Individualized Tumor Therapy'. Peter Fasching emphasized the potential of Germany as a leader in the conduction of clinical trials and within them the asservation of biomaterials along with clinical patient data. As it has become tradition, the symposium gave young researchers the opportunity to present their innovative translational research strategies and models. Out of numerous abstracts, the best 4 were selected for oral presentation. As a new component in the educational package offered by the TraFo symposium, tutorials by experienced scientists and statisticians covering themes such as statistics, study design, publishing, presentation and molecular analysis and lab work served to equip aspiring researchers with the tools for success.

\section{COMBATing 2014}

Following the TraFo Meeting, COMBAT started off with a warm welcome from Nadia Harbeck. The first session covered the most recent developments since the previous conference. Jürgen Dittmer (University Hospital Halle) introduced the audience to new insights into the importance of interaction between tumor cells and surrounding tissue, demonstrating the effect of stromarich tumor histomorphology on prognosis and detailing current hypotheses on the effects of fibroblast activation and stromal glycolysis activity. An overview over current clinical trials evaluating and comparing classic chemotherapy, endocrine, and HER2targeted therapeutic concepts to avoid over- and undertreatment in primary breast cancer was given by Oleg Gluz (Bethesda Hospital Mönchengladbach) for the Women's Healthcare Study 
Group (WSG). The wealth of markers used to categorize and stratify tumors necessitates innovative concepts to keep clinical trials feasible, such as the evaluation and establishment of surrogate parameters including disease-free survival and complete histopathological remission rate. Apart from the introduction of new substances, trials seek to optimize choice, sequence, density, and intensity of therapy and to custom tailor it for each patient, using, identifying, and validating new biomarkers. Current estimations suggest that about 35 mutations separate a cancer cell from a healthy cell, and each of these may be a potential prognostic and predictive factor or even a therapeutic target. As a prominent example, Peter Fasching's lecture described the PI3K pathway, from PI3K's physiological functions to pathological alterations in oncogenesis to its modulation as a therapeutic concept and associated challenges concerning patient selection and toxicity management. It is representative of the process of 'digging deeper' into the complex mechanisms regulating mitotic activation and inhibition and cell cycle processes within the cell and in the context of its microenvironment. As a later step in pro-mitotic signaling, cycline dependent kinases were discussed by Cornelia Liedtke (University Hospital Schleswig-Holstein Campus Lübeck), who demonstrated the potential of their inhibition and upcoming targeted agents, with the most advanced in clinical trials being palbociclib. In the last lecture of this session, Günter Steger (Medical University Vienna) outlined new additions to the breast cancer drug arsenal, with a focus on trastuzumab emtansine as one of the few truly new agents, and underlined the international heterogeneity in handling of drug approval.

For the first time, the COMBATing agenda included a separate session on eHealth. Timo Schinköthe (University Hospital Munich), representing Germany, and Andreas Trojan (OncoCenter Zurich), representing Switzerland, provided an exciting and informative look into this innovative field of medical science apart from traditional patient care. In Switzerland and Germany, the assistance of oncologic patient care via internet or mobile device was shown to be well accepted across various patient demographic groups including elderly patients, with programs such as Cankado (www.cankado.de) or Consilium showing great developmental maturity and promising to facilitate therapy monitoring and patient guidance. Both presentations showed that the concepts currently employed in routine care are just the tip of the iceberg, with major companies currently investing massively in advancing this branch of medical care.

The third session provided an opportunity for the conference's industry sponsors to demonstrate the latest and most advanced products in their respective pipelines. Eugen Ruckhäberle (University Hospital Düsseldorf) showed the history of the development of Astra Zeneca's olaparib as the best-known representative of PARP inhibitors. He outlined the promises of early trials focusing on the treatment of breast and ovarian cancer and the setbacks suffered before this substance reemerged in the more narrow setting of BRCA-mutated breast cancer. Maik Pruess of NanoString presented the standardized version of the PAM50 test called Prosigna, and its applications and potential in clinical deci- sion-making, providing evidence-based guidance in formerly unclear situations, and as a new research tool in multigenetic testing. Lastly, Gernot Guderian of Novartis explained the design of the BELLE and MONALEESA trials investigating the previously introduced PI3K and CDK4/6 inhibitors in the setting of metastatic breast cancer and the networks needed to conduct these new trials.

A highlight of the conference was the Award Lecture (HennerGraeff Award 2012) held by Georg Pfeiler, a young professor of Medical University Vienna, on the difficulties and pitfalls of development of targeted therapies and selection and validation of markers predictive of therapeutic efficacy. Recent results of his research suggest an impact of BMI and body fat on the effectiveness of endocrine therapy with different agents.

The day concluded with the laudation for Frederik Marmé and the announcement of the winners of the COMBAT travel scholarship, Eva-Beatrice Kohls, Kim Chi Katharina Ho (University Hospital Schleswig-Holstein Campus Kiel), Florian Schindler (Hospital Starnberg), Lennard Schröder, and Julian Koch (University Hospital Munich).

The second day started off with the Key Note Lecture held by Bruce A. Littlefield of Eisai on drug development using eribulin as an example, both as a molecule and a therapeutic agent. He reminded the audience that 'there is no history of success in medicine, but a history of mistakes made and lessons learnt', as Eribulin's complex chemical structure made its synthesis an unusual challenge. Its remarkable effect on overall survival in the metastatic breast cancer setting encourages detailed exploration of its mechanisms of action. Hypotheses concerning effects apart from traditional antimitotic activity include tumor vasculature remodeling, reversal of epithelial-mesenchymal transition, and induction of decreased capacity for migration and invasion.

The session on signaling pathways commenced with a lecture by Christian Singer (Medical University Vienna) focused on the role of RANK signaling both in bone metastasis and in breast tumorigenesis, thereby providing a possible explanation for the increase in breast cancer risk associated with progesterone-containing hormone replacement therapy. Further away from generating an effective clinical treatment approach but nevertheless highly intriguing, the Src pathway was explained by Beyhan Ataseven (Hospital Essen-Mitte). We currently lack sufficiently predictive biomarkers to identify a patient population that may benefit from the use of Src inhibitors (such as dasatinib) in breast cancer in order to offset their toxicity profile, as well as an ideal combination partner substance. The session was concluded by Volkmar Müller's (University Hospital Hamburg-Eppendorf) comparison of circulating tumor cells and circulating tumor DNA. The data quality concerning prognostic value favors CTCs, but opportunities for application of both methods in the clinical setting are continuously refined as clinical trials (such as the ambitious DETECT trial series) aim to clarify their predictive potential and establish them as biomarkers to guide therapeutic choices. 
The next topic was dedicated to hereditary and triple negative breast cancer (TNBC). Kerstin Rhiem (University Hospital Cologne) provided an outlook on intermediate- and low-risk mutations apart from the well-established $B R C A$ defects. Given the vast spectrum of mutations and their respective clinical relevance, she illustrated the need for effective tools for individual risk assessment and for clear guidance to aid clinical decision-making regarding the use of prophylactic diagnostics and procedures. She also presented the new guidelines by AGO and the German Consortium on Hereditary Breast and Ovarian Cancer. Preventive strategies and their application in high-risk and intermediate-risk mutation carriers were discussed by Nina Ditsch (University Hospital Munich), separated into primary (before onset of disease), secondary (early detection), and tertiary (in diagnosed patients) forms of prevention. She summarized that risk-adapted screening should be applied to affected women. Furthermore, she underlined the effectiveness of prophylactic adenectomy and mastectomy in both BRCA1 and BRCA2 mutation carriers while warning of a too liberal use of these invasive procedures in women with a low or unclear risk profile. Apart from hereditary factors, other carcinogenic influences such as radiation exposure may warrant the use of intensified preventive measures. New guidelines for $B R C A$ mutation carriers are under development and will be presented in early 2015.

Cornelia Liedke offered a detailed look into data regarding the use of platinum-based agents in BRCA-positive and in any TNBC. She concluded that platinum based chemotherapy may offer improved survival in TNBC at the cost of significant toxicity, though currently available data from trials such as GeparSixto (carboplatin vs. control, combined with paclitaxel and NPLD) and CALBG40603 (carboplatin vs. control, combined with paclitaxel) are limited to $\mathrm{pCR}$ rates with survival analysis event thresholds still on the horizon. In a different approach, the ADAPT TN sub-study randomizes carboplatin + nab-paclitaxel vs. gemcitabine + nab-paclitaxel, thereby deescalating instead of intensify- ing the therapy. While the AGO guidelines offer to recommend platinum therapy for any TNBC, trial participation is still preferred since more mature data as well as better validated predictive tools are required to guide patient selection for these therapies. Candidates for predictors include gene signatures, individually or summarized as a homologous recombination deficiency score (HRD), as well as microenvironment-oriented features such as tumor infiltrating lymphocytes.

The final session centered on multigene assays. Their prognostic potential was laid out by Marcus Schmidt (University Hospital Mainz), who also presented the current AGO guidelines regarding their use for patient selection for adjuvant therapy and emphasized the increasing wealth of supporting data. He also provided background information to decide their reliability compared to basic immunohistochemistry for ER/PR/HER2/Ki67 evaluation. Michael Knauer (Hospital of the St. Gallen) elaborated on predictive applications of the assays. The more established tests - OncotypeDX, EndoPredict, and PAM50 - are recognized by guidelines as useful tools in the subset of clinical situations where indication based on traditional criteria remains unclear. In trials, about $30 \%$ of clinical decisions were altered based on test results. Apart from systemic therapy, Eugen Ruckhäberle explained how also loco-regional treatment issues ranging from surgical margins to radiotherapy may receive guidance from such tests using tools such as the genomic nodal index predicting nodal status, which may help reduce or entirely avoid nodal surgery in low-risk patients.

In summary, COMBAT was again extraordinarily successful in furthering the exchange of information between lab-based research and clinical practice as well as in teaching doctors and scientists about breast cancer and its treatment. The annual meeting tradition is scheduled to continue in fall of 2015.

Further information on COMBAT is available at www.combating.de alongside many original presentations and materials.

J.G. Koch , L. Schroeder , R. Wuerstlein 